American Journal of Pharmacology and Toxicology 1 (4): 79-82, 2006

ISSN 1557-4962

(C) 2006 Science Publications

\title{
Rapamycin Inhibits Cystogenesis by Cystic Epithelial Cells Derived from Human Autosomal Dominant Polycystic Kidneys
}

\author{
${ }^{1}$ Gerard Elberg, ${ }^{2}$ Teresa V. Lewis, ${ }^{1}$ Lijuan Chen and ${ }^{1}$ Martin A. Turman \\ ${ }^{1}$ Department of Pediatrics, University of Oklahoma Health Sciences Center \\ Oklahoma City, Oklahoma, USA \\ ${ }^{2}$ College of Pharmacy, University of Oklahoma Health Sciences Center, Oklahoma City, Oklahoma, USA
}

\begin{abstract}
Autosomal dominant polycystic kidney disease (ADPKD) is one of the most common genetic disorders and carries a high health burden since it progresses to renal failure in half of the afflicted individuals by the $6^{\text {th }}$ decade of life. Currently, there is no effective treatment to slow the progression of ADPKD. Rapamycin, a drug most commonly used as an immunosuppressive agent for organ transplantation, slows cystogenesis in several rodent models of polycystic kidney disease; however, its efficacy in human ADPKD is unknown. We tested the ability of rapamycin to inhibit cyst formation in an in vitro model of ADPKD using cystic epithelial cells derived from the cyst walls of kidneys from patients with ADPKD. Cystic epithelial cells were cultured in a 3-dimensional matrix of Matrigel and collagen I. In the absence of rapamycin, numerous large cystic structures formed when visualized by phase-contrast microscopy. Rapamycin decreased cyst size and cyst number in a dose-dependent manner. The concentration of rapamycin that decreased cyst number by $50 \%\left(\mathrm{IC}_{50}\right)$ was $1.4 \pm 0.2 \mathrm{nM}$, a level achievable with current dosing regimens in transplantation. Staining nuclei with DAPI and then examining the gels with an inverted fluorescent microscope further visualized cell structures. This revealed that rapamycin was not toxic to cystic epithelial cells and supported the formation of branching tubule-like structures in the 3-dimensional gels. This study demonstrates that rapamycin inhibits cystogenesis by human ADPKD cystic epithelial cells. Rapamycin is a potent immunosuppressive agent, which has many potential side effects. This study provides further impetus to subject otherwise healthy individuals at the early stage of ADPKD to many years of rapamycin in a prospective clinical trial.
\end{abstract}

Key words: polycystic kidney disease, rapamycin, human, 3-dimensional cultures

\section{INTRODUCTION}

Autosomal dominant polycystic kidney disease (ADPKD) affects 1 in a 1,000 individuals, making it one of the most common genetic disorders ${ }^{[1]}$. ADPKD progresses to renal failure in half of the affected individuals by age 60 and is a common cause of end-stage renal disease ${ }^{[2]}$. There is currently no effective treatment to slow the progression of ADPKD.

Most cases of ADPKD are caused by mutations in polycystin-1 (PC-1) $)^{[2]}$. PC-1 is a large protein (520 $\mathrm{kDa}$ ) with a relatively short cytoplasmic tail ${ }^{[2]}$. The cytoplasmic tail of PC-1 associates with mTOR (mammalian target of rapamycin) and mTOR is anomalously overexpressed in cystic epithelial cells of ADPKD patients ${ }^{[3]}$. Furthermore, rapamycin, an inhibitor of mTOR, decreased cystogenesis and improved renal function in the orpk-rescue and the bpk mouse models of $\mathrm{PKD}^{[3]}$. Rapamycin also reduced cyst formation in the Han:SPRD rat model of PKD ${ }^{[4]}$. The mutations causing PKD in these models are distinct from the mutations causing ADPKD; however, mutations in many different "cystogenic" genes are in some way associated with the single primary cilia found on renal tubular epithelial cells ${ }^{[1,5]}$. Mostov ${ }^{[6]}$ hypothesized that ciliary dysfunction is a common pathway leading to loss of regulation of mTOR with subsequent unregulated activation of mTOR. Activated mTOR stimulates various downstream signals, such as S6 kinas $^{[6]}$. The mechanism by which this overactivation leads to cyst formation is not clear, but inhibition of mTOR with rapamycin increases apoptosis in murine cystic epithelial cells ${ }^{[3]}$, which suggests that regulation of apoptosis plays an important role in cyst formation.

Corresponding Author: $\quad$ Martin A. Turman, MD, PhD, Pediatric Nephrology, 940 NE $13^{\text {th }}$ Street 2B2309, Oklahoma City, OK 73034, Tel: 405-271-4409, Fax: 405-271-3967 
Rapamycin is clinically approved as an immunosuppressive drug for organ transplantation. Shillingford et $a{ }^{[3]}$ retrospectively analyzed patients with ADPKD who had received a kidney transplant and who had a renal CT scan before and approximately 2 years after transplant. The patients on a rapamycinbased immunosuppressive regimen had a greater decrease in kidney volume over 24 months than the patients on other regimens. This provides indirect evidence that rapamycin may be effective in humans for the treatment of ADPKD.

\section{MATERIALS AND METHODS}

Cell cultures: Cystic epithelial cells (CEC) from the cyst walls of human kidneys from two individuals with autosomal dominant polycystic kidney disease (ADPKD) were isolated essentially as described ${ }^{[7]}$.

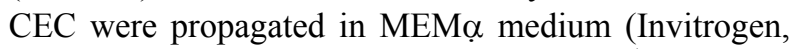
Carlsbad, CA) supplemented with 10ng $\mathrm{mL}^{-1} \mathrm{EGF}, 5 \mu \mathrm{g}$ $\mathrm{mL}^{-1}$ hydrocortisone (Sigma, St. Louis, MO), 6.25 $\mu \mathrm{g}$ $\mathrm{mL}^{-1}$ insulin, $6.25 \mu \mathrm{g} \mathrm{mL}^{-1}$ transferin, 6.25ng $\mathrm{mL}^{-1}$ selenous acid (BioWhittaker, Walkersville, ME), 10\% $(\mathrm{v} / \mathrm{v})$ heat inactivated fetal bovine serum (Atlanta Biologicals, Atlanta, GA) and 1\% antibiotic/antimycotic solution (Invitrogen).

Three dimensional gel system: CEC $\left(0.5 \times 10^{5}\right.$ cells/well) were suspended in a $4^{\circ} \mathrm{C}$ solution containing complete MEM $\alpha$ and a 1:1 (v/v) mixture of liquid rat tail collagen I and Matrigel (Becton Dickinson, Franklin Lakes, NJ) and poured into cell culture inserts as previously described ${ }^{[8]}$. Cells were cultured in growth medium as above supplemented with $77 \mathrm{nM}$ $\mathrm{PGE}_{2}$ (Sigma) and different concentrations of rapamycin (LC labs, Woburn, MA) indicated in figure legends. Culture medium was replaced every 2 days and cells were cultured for 14 days. Cysts were viewed with an IX50 inverted phase contrast and fluorescence microscope (Olympus, Melville, NY). Cyst size was determined by measuring the planar area of cysts using SPOT imaging software (Diagnostic Instruments, Sterling Heights, MI). A cyst was defined as a circular cell structure with a minimum planar area of 5,000 $\mu^{2}$. To visualize nuclei, cells were cultured for an additional day and then fixed by incubating gels in $10 \%$ formalin (Labsco, Louisville, KY) for 40 minutes. Cells were then permeabilized with $0.2 \%$ Saponin in phosphate-buffered saline (PBS) supplemented with 1\% BSA (Sigma) for 1hour. Nuclei were stained by incubation with $10 \mu \mathrm{g} \mathrm{mL}^{-1}$ 4'-6-Diamidino-2- phenylindole (DAPI, Sigma) in PBS for 1 hour. After washing in PBS, DAPI-stained nuclei were observed by fluorescence microscopy.

Inhibition curve and statistical analysis: Data processing, inhibition curve analysis and statistical analysis was performed using GraphPad software (San Diego, CA). The relationship between rapamycin dose and cyst size was analyzed by non-parametric ANOVA with Kruskal-Wallis test and post-hoc analysis with Dunn's Multiple Comparison Test.

\section{RESULTS}

Rapamycin inhibits cyst formation: To determine if rapamycin inhibits cyst formation by $\mathrm{CEC}$ derived from human ADPKD kidneys, a 3-dimensional culture system was utilized as a model of human ADPKD. CEC cultured in the collagen:Matrigel mixture formed multiple large cysts (Fig. 1). Addition of increasing concentrations of rapamycin to $\mathrm{CEC}$ cultures resulted in a dose-dependent reduction in cyst size and number (Fig. 1-3). Analysis of cyst size demonstrated a decrease with increasing concentrations of rapamycin (Fig. 2). Cyst sizes in all concentrations of rapamycin were significantly less than in the absence of rapamycin $(p<0.05)$. At higher doses of rapamycin very few discernable cysts formed and those that did form were much smaller than in the absence of rapamycin. The formation of large cysts was abolished by rapamycin. Fig. 3 demonstrates that rapamycin decreased the number of cysts that formed in 3-dimensional cultures. The total number of cysts $>5,000 \mu \mathrm{m}^{2}$ in 25 fields of view was $447,514,341,119,26$ and 16 with treatment with $0,0.5,1.0,2.5,5.0$ and $10 \mathrm{nM}$ rapamycin, respectively. This data was used to determine that the concentration of rapamycin resulting in a 50\% reduction in cyst number $\left(\mathrm{IC}_{50}\right)$ was $1.4 \pm 0.2 \mathrm{nM}$ (Fig. 3).

Rapamycin is not toxic to $\mathrm{CEC}$ and supports the formation of tubule-like structures: We used DAPI staining of nuclei to visualize cells dispersed throughout the 3-dimensional cultures (Fig. 4). An abundant number of nuclei could be seen in cultures incubated with rapamycin by fluorescent microscopy of DAPI-stained culture. This result indicates that the lack of large cysts visualized by phase contrast microscopy in the presence of rapamycin was not due to loss of CEC from rapamycin toxicity. In the presence of rapamycin, $\mathrm{CEC}$ also formed some tubule-like branching structures, providing further confirmation 


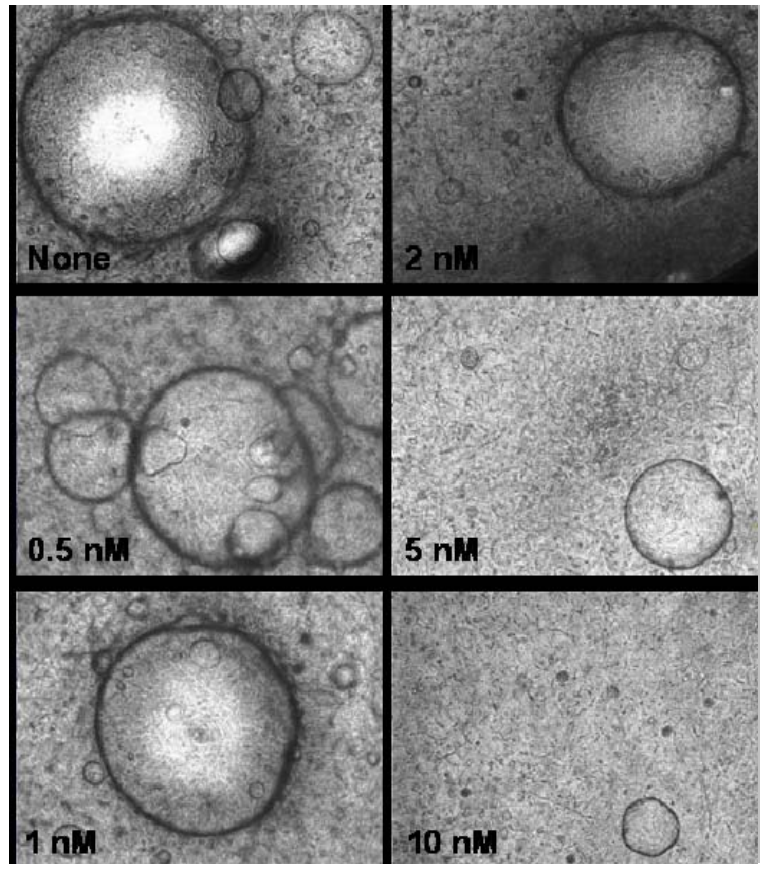

Fig. 1: Effect of rapamycin on cyst formation. CEC cultured in collagen/Matrigel were treated with different concentrations of rapamycin as indicated on each picture. Pictures depict the appearance by phase contrast microscopy of the largest cyst visualized for each treatment after 14 days at magnification $\mathrm{X} 40$

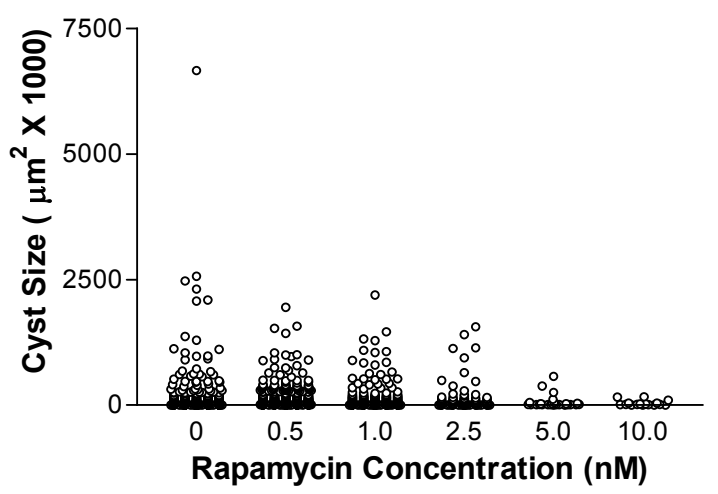

Fig. 2: Effect of rapamycin on cyst size. CEC were cultured in collagen/Matrigel with varying concentrations of rapamycin for 14 days. Cyst size was then determined from pictures taken of 25 different fields of view at $40 \mathrm{X}$ magnification. Data is pooled from CEC derived from 2 different donors. Cyst sizes in all concentrations of rapamycin were significantly less than in the absence of rapamycin $(\mathrm{p}<$ $0.05)$

that rapamycin was not toxic to CEC. Therefore, rapamycin inhibits cystogenesis, but not tubulogenesis, which indicates a specific effect on inhibiting cyst formation.

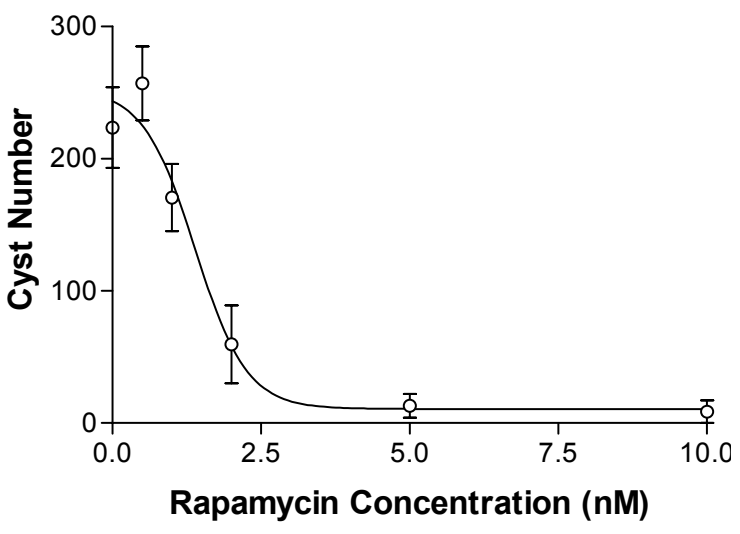

Fig. 3: Analysis of the effect of rapamycin on cyst number. CEC were cultured in collagen/Matrigel with varying concentrations of rapamycin for 14 days. Cyst number was then determined from pictures taken of 25 different fields of view at $40 \mathrm{X}$ magnification. $\mathrm{IC}_{50}$ was $1.4 \pm 0.2 \mathrm{nM}$ as determined using GraphPad software analysis. Data is pooled from $\mathrm{CEC}$ derived from 2 different donors

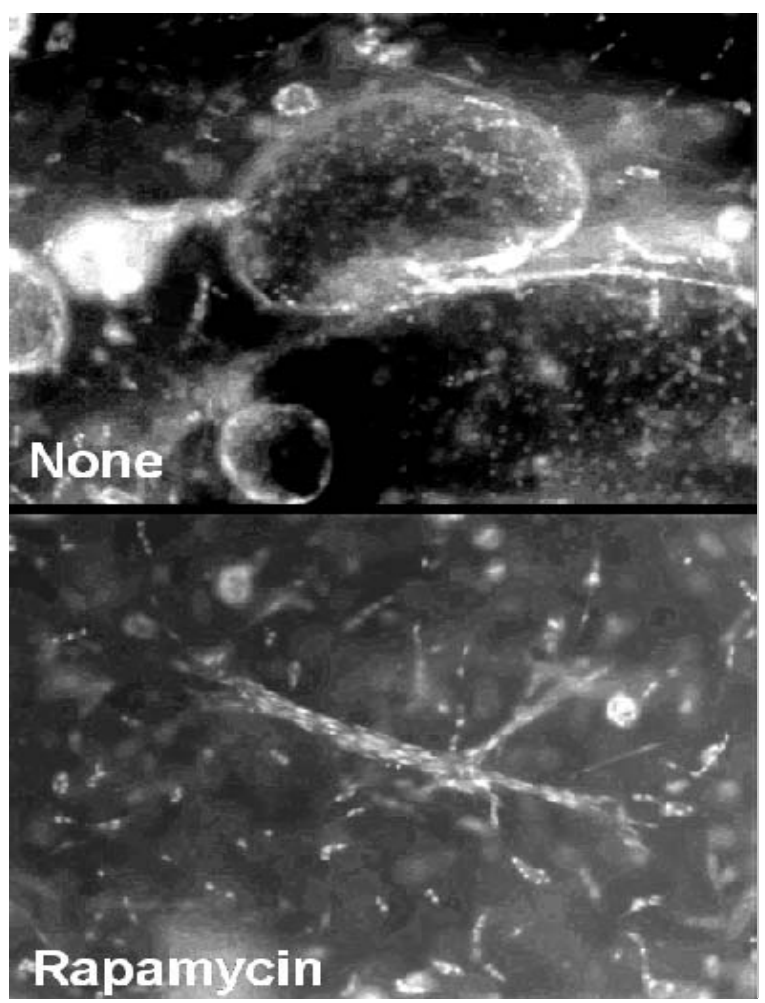

Fig. 4: Analysis of rapamycin effect on cyst formation and cell viability. CEC in collagen/Matrigel gel were grown in the presence or absence of $10 \mathrm{nM}$ rapamycin for 15 days. Cells were then fixed and analyzed with DAPI staining and fluorescence microscopy at magnification X100. Nuclei stained with DAPI demonstrate inhibition of cyst formation in the presence of rapamycin but sustained cell viability. In the presence of rapamycin some tubule-like structures formed 


\section{DISCUSSION}

Previous studies demonstrated that rapamycin inhibits cystogenesis in several animal models of $\mathrm{PKD}^{[3,4]}$. Although there may be a common mechanism underlying cyst formation in all forms of PKD, there may be distinct differences between rodent models of PKD and human ADPKD since many drugs that are efficacious in rodent models turn out to be ineffective in humans. In this study we report that rapamycin can inhibit the formation of cysts in a 3-dimensional gel culture system utilizing human CEC derived from patients with ADPKD. The fact that rapamycin greatly decreases cyst size and number in this study is consistent with the striking results obtained in murine models of $\mathrm{PKD}^{[3,4]}$. This study supports the notion that rapamycin will slow the progression of ADPKD in humans and provides further incentive to begin clinical trials with rapamycin for the treatment of ADPKD.

When rapamycin was given to animals with PKD, they received 2 to $5 \mathrm{mg} \mathrm{kg}^{-1}$ per day of drug ${ }^{[3,4]}$. After renal transplantation, patients usually receive 1 to 10 $\mathrm{mg}$ per day of rapamycin and dosage is adjusted by whole blood trough concentrations with the goal usually being 5 to $20 \mathrm{ng} \mathrm{mL}$. This corresponds to a much lower $\mathrm{mg} / \mathrm{kg}$ dose in humans than that used for the animal studies. In our study, we found that the $\mathrm{IC}_{50}$ to inhibit cyst formation was $1.4 \mathrm{nM}$, which corresponds to a concentration of $1.3 \mathrm{ng} \mathrm{mL}^{-1}$. This suggests that the dose used in organ transplantation may be therapeutic in slowing cyst formation in patients with ADPKD.

Shillingford et al. ${ }^{[3]}$ demonstrated that rapamycin accelerated the loss of volume of native ADPKD kidneys after renal transplantation. However, ADPKD patients that require a kidney transplant have end-stage kidney disease and the native ADPKD kidneys are essentially non-functional. Although intriguing, the relevance of the rate of decrease in kidney volume after transplantation is not clear. It is not known if this size difference corresponds to reversal of cyst formation and improved function of the native kidneys or represents a less significant change. Treatment of ADPKD patients with rapamycin will require years of treatment before the onset of end-stage renal disease for success. However, rapamycin has many untoward side effects, such as increased risk for infections, problems with wound healing, hypertension, edema, proteinuria, hyperlipidemia and anemia ${ }^{[9]}$. This study provides evidence that rapamycin can slow cyst formation in human CEC. Thus, this study provides further impetus to subject otherwise healthy individuals at the early stage of ADPKD with rapamycin for years in a prospective clinical trial.

\section{ACKNOWLEDGEMENTS}

We gratefully acknowledge the support of the Polycystic Kidney Disease Foundation, the Children's Medical Research Institute and Children's Miracle Network Foundation.

\section{REFERENCES}

1. Guay-Woodford, L.M., 2006. Renal cystic diseases: diverse phenotypes converge on the cilium/centrosome complex. Pediatr. Nephrol., 21: 1369-1376.

2. Wilson, P.D., 2004. Polycystic kidney disease. N. Engl. J. Med., 350: 151-164.

3. Shillingford, J.M., N.S. Murcia, C.H. Larson, S.H. Low, R. Hedgepeth, N. Brown, C.A. Flask, A.C. Novick, D.A. Goldfarb, A. Kramer-Zucker, G. Walz, K.B. Piontek, G.G. Germino and T. Weimbs, 2006. The mTOR pathway is regulated by polycystin-1 and its inhibition reverses renal cystogenesis in polycystic kidney disease. Proc. Natl. Acad. Sci. USA, 103: 5466-5471.

4. Wahl, P.R., A.L. Serra, H.M. Le, K.D. Molle, M.N. Hall and R.P. Wuthrich, 2006. Inhibition of mTOR with sirolimus slows disease progression in Han:SPRD rats with autosomal dominant polycystic kidney disease (ADPKD). Nephrol. Dial. Transplant., 21: 598-604.

5. Zhang, Q., P.D. Taulman and B.K. Yoder, 2004. Cystic kidney diseases: all roads lead to the cilium. Physiology (Bethesda), 19: 225-230.

6. Mostov, K.E., 2006. mTOR is out of control in polycystic kidney disease. Proc. Natl. Acad. Sci. USA, 103: 5247-5248.

7. Wallace, D.P., J.J. Grantham and L.P. Sullivan, 1996. Chloride and fluid secretion by cultured human polycystic kidney cells. Kidney Int., 50: 1327-1336.

8. Guruswamy, S., S. Lightfoot, M.A. Gold, R. Hassan, K.D. Berlin, R.T. Ivey and D.M. Benbrook, 2001. Effects of retinoids on cancerous phenotype and apoptosis in organotypic cultures of ovarian carcinoma. J. Natl. Cancer Inst., 93: 516525.

9. Kuypers, D.R., 2005. Benefit-risk assessment of sirolimus in renal transplantation. Drug Saf, 28: 153-181. 